\title{
Direct Detection of DNA on Gold Structured Planar Substrates by Raman microscopy
}

\author{
F Carpignano $^{1,2}$, J A Grant-Jacob ${ }^{1}, \mathrm{~J} \mathrm{Lamb}^{3}, \mathrm{~K}$ Pechstedt $^{1}$, W S Brocklesby ${ }^{1}$, T Melvin ${ }^{1,3^{*}}$ \\ ${ }^{1}$ Optoelectronics Research Centre, University of Southampton, UK \\ ${ }^{2}$ Dipartimento di Ingegneria Industriale e dell'Informazione, Università di Pavia, Italy \\ ${ }^{3}$ Electronics and Computer Science, University of Southampton, UK
}

\begin{abstract}
Detection of DNA sequences is pivotal to many modern molecular diagnostic methods, but the ability to directly detect DNA sequences, without the need for signal amplification (such as by applying a polymerase chain reactions) is highly desirable. Here we investigate the potential for gold inverted pyramidal structures (also known as Klarite ${ }^{\circledR}$ ) for DNA detection, as these simple chips containing the structured surface on a face could offer an improved format for DNA based diagnostic methods. Our strategy included optimization of the fabrication protocols to achieve flat gold surfaces within the inverted pyramidal surface and then a subsequent assessment of these substrates for direct DNA detection by Raman microscopy. These studies demonstrate for the first time the potential of these gold structured planar substrates for DNA analysis applications.
\end{abstract}

\section{INTRODUCTION}

Although Raman spectroscopy offers an approach for fingerprint identification of molecules, the extremely weak Raman cross section means that the detection of low concentrations of bioanalytes is impossible. An enhancement of cross section can be achieved when the molecules are in close proximity to a metal surface of nanoscale roughness, providing surface enhanced Raman scattering (SERS) and surface enhanced resonant Raman scattering approaches for improved sensitivity for bioanalyte detection. [1] Most research has been focused on colloidal structures,[2] lithographically designed arrays [3] or nanoscale motifs patterned on substrates. [4] For most of these structures the broad plasmon resonance is tuned as a function of the shape or separation.

Metallic microcavities or voids offer a better alternative as photons are coupled into these structures yielding localised plasmon cavities whereby more optimal surface-enhanced Raman scattering is observed.[5] Using this more recent strategy there has been a significant effort to produce substrates suitable for SERS based diagnostic methods, notably by the design of mesostructured metallic materials. This has built upon a greater understanding of the plasmonic processes within these structures that underpin the enhancements. As a result there has been an emergence of metallic structure designs where both localised plasmons as well as a strong coupling to external light is achieved. [6-10]

Of the designs considered for SERS applications, inverted pyramidal substrates (also known as Klarite $\AA$ ) are well understood with respect to optimal cavity size to achieve the so called 'resonant plasmon cavity'.[6, 10] The inverted pyramidal structures are created by an anisotropic $\mathrm{KOH}$ etch of (100) orientated silicon wafers with square apertures aligned along the [100] direction. Due to preferential etching along the $\{111\}$ planes atomically smooth and reproducible substrates are created which can be covered with a metallic surface. Highly smooth gold surfaces coating over the atomically smooth silicon faces are considered as optimal for creating the 'resonant plasmon cavity', where the polaritons are assumed to oscillate up and down the sides of the inverted pyramid and are reflected by the sharp inverted pyramid edge. Where the gold surface is in close contact with an analyte, the plasmons then transfer energy to analytes and following the Raman process the energy is transferred back to the plasmon less the amount transferred to the analyte nuclei and scattered from the surface. Although resonant plasmon cavities have clear advantages for SERS applications (notably the $>10^{6}$ SERS enhancement and the reproducibility (better than 10\%)[6]) plasmon interference within the resonant cavity must be considered, in other words the outgoing and ingoing resonances must be tuned for the pyramid cavity size, and the correct cavity size must be used for a given pump wavelength (and SERS signals). Inverted pyramid cavities with a period of $2000 \mathrm{~nm}$ and an aperture size of $1220 \mathrm{~nm}$ have been shown to the most effective geometry to couple white light ( $\sim 70 \%$ absorption), showing strong resonant absorption at $\sim 800 \mathrm{~nm}$. [5] The strong absorption is considered indicative of the strength of the electric field on the gold surface and vital for SERS.

DNA nucleic acid bases in DNA sequences have been detected directly using metallic nanoparticles and nanoshells and other substrates by SERS approaches.[2] We considered the inverted pyramidal structures in a planar substrate (Figure 1) as a simpler assay format for diagnostic applications. In addition these inverted pyramidal structures have not been fully investigated with respect to direct DNA nucleic acid base detection.

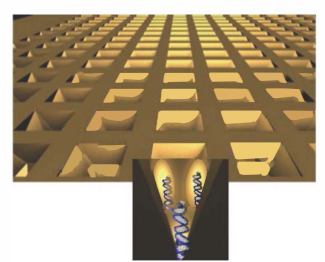

Fig. 1. Schematic of DNA inside gold coated pyramidal cavity structures. 


\section{MATERIALS AND METHODS}

\section{A. Fabrication of SERS substrates}

Silicon substrates with inverted pyramid pits were kindly provided by Rennishaw Diagnostics, Glasgow, UK. The substrates contain an array of inverted pyramids etched into a series of $4 \mathrm{~mm} \times 4 \mathrm{~mm}$ square regions on the wafer with flat borders; the wafers are diced into rectangular chips of approximately $1.2 \mathrm{~cm} \times 0.8 \mathrm{~cm}$. The inverted pyramid design used is $1500 \mathrm{~nm} \times 1500 \mathrm{~nm}$ square, $1000 \mathrm{~nm}$ deep and with a pitch of $2000 \mathrm{~nm}$. The pyramid faces are oriented at an inclination of $35.3^{\circ}$ to the normal. A BOC Edwards Auto E500a Electron Beam Evaporator was used to evaporate a $5 \mathrm{~nm}$ thick layer of chrome onto the surface. Following the deposition of the chrome, a $100 \mathrm{~nm}$ thick layer of gold was evaporated onto the substrate either by using an Edwards E306A Thermal Evaporator or a BOC Edwards Auto E500a Electron Beam Evaporator. The samples were then evaluated using an Orion ${ }^{\circledR}$ Plus helium ion microscope (HIM).

\section{B. Oligonucleotide synthesis}

The oligonucleotides shown in Table 1 and the monodeoxynucleotides $\mathrm{dA}_{10}, \mathrm{dG}_{10}, \mathrm{dT}_{10}$ and $\mathrm{dC}_{10}$ were synthesized as previously reported.[5] Dithiol phosphoramidites where purchased from Glen Research Inc. To incorporate Texas Red chromophore at the 3'-end of the C7-aminoalkyl oligonucleotide, $50-150 \mathrm{nmol}$ of the oligonucleotide in $70 \mu \mathrm{l}$ of $0.5 \mathrm{M} \mathrm{Na} \mathrm{CO}_{3} / \mathrm{NaHCO}_{3}$ buffer $(\mathrm{pH}$ 8.75 ) for $4 \mathrm{~h}$ at room temperature with $1 \mathrm{mg}$ of the Texas Red succinimide ester (Invitrogen). The Texas Red-labelled oligonucleotides were purified by reverse-phase HPLC (ABI Aquapore column (C8) $8 \mathrm{~mm} \times 250 \mathrm{~mm}$, pore size $300 \AA(0.1 \mathrm{M}$ (A) ammonium acetate ( $\mathrm{pH}=7)$, (B) $0.1 \mathrm{M}$ ammonium acetate in acetonitrile $(50 \%)$ with increase in the gradient concentration of $\mathrm{A}: \mathrm{B}$ to $60 \%$ for the elution of the two isomeric Texas red-labelled oligonucleotides separately and then subsequent desalting using a NAP-10 elution column (GE Healthcare).

TABLE I. OLIGONUCLEOTIDE SEQUENCES

\begin{tabular}{|l|r|}
\hline Probe & XHXHXHGCAGCAAATTGCACTGGAGTGCGAG-3'-TR ${ }^{\mathrm{a}, \mathrm{b}}$ \\
Target & 3'-GTGACCTCACGCTC-5' - TR $^{\mathrm{b}}$ \\
\hline
\end{tabular}

${ }^{\mathrm{a} .}$ Where $\mathrm{X}$ is a disulfide monomer and $\mathrm{H}$ is a hexaethyleneglycol spacer[5

$$
\text { b. Where TR is Texas red fluorophore }
$$

\section{Immobilization and subsequent hybridisation of} oligonucleotides on the substrate.

The dithiol-modified oligonucleotide (Probe, Table 1) was diluted to $1 \mu \mathrm{M}$ concentration with $\mathrm{pH} 8.1$ phosphate buffer containing $0.1 \mathrm{M} \mathrm{NaCl}$. For immobilizing the probe oligonucleotide, the $4 \mathrm{~mm} \times 4 \mathrm{~mm}$ square region of several of the gold pyramidal substrates was coated with $100 \mu \mathrm{L}$ of the solution of the oligonucleotide and incubated at $6{ }^{\circ} \mathrm{C}$ for $48 \mathrm{~h}$. After this, the substrates were taken out and washed with phosphate buffer containing $0.1 \mathrm{M} \mathrm{NaCl}$. (pH 8.1) and dipped in $10 \mu \mathrm{M}$ mercaptohexanol solution prepared in phosphate buffer containing $0.1 \mathrm{M} \mathrm{NaCl}$. (pH 8.1) for 20 min (Figure 2).
The substrates were taken out and rinsed with phosphate buffer containing $0.1 \mathrm{M} \mathrm{NaCl}$. ( $\mathrm{pH} \mathrm{8.1)} \mathrm{and} \mathrm{then} \mathrm{deionised}$ water and dried with filtered nitrogen.

The Texas red labelled target oligonucleotide was diluted to a concentration of $1 \mu \mathrm{M}$ in $10 \mathrm{mM}$ TRIS buffer ( $\mathrm{pH} 7)$ and $100 \mu \mathrm{L}$ of $1 \mu \mathrm{M}$ oligonucleotides were added to the surface of the inverted pyramid substrates that had previously been functionalized with the probe oligonucleotide (Figure 2). The substrates were incubated overnight at $6{ }^{\circ} \mathrm{C}$ and then washed thoroughly with deionized water and then dried with filtered nitrogen.

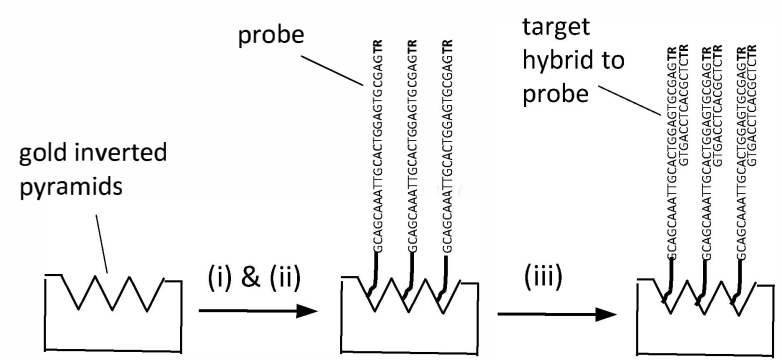

Fig. 2. Schematic of the protocol for DNA functionalization. (i) Addition of the probe oligonucleotide (Table I), (ii) addition of a solution of mercaptohexanol to prevent non-specific binding, (iii) hybridization of the complementary Target oligonucleotide (Table I)

\section{Raman microscopy analysis}

Spectra were acquired using a Renishaw inVia Raman system with a Leica microscope (Leica N Plan $50 \times$ and Leica N Plan EPI $20 \times$ objectives (NA: 0.75 and 0.40 respectively)). A $785 \mathrm{~nm}$ diode laser is integrated in the inVia system. Raman spectra were acquired from a $60 \mu \mathrm{m} \times 60 \mu \mathrm{m}$ or larger area on the substrate with the laser being moved approximately $20 \mu \mathrm{m}$ each time. The spectra were acquired for $10 \mathrm{~s}$, under either static mode centred at $520 \mathrm{~cm}^{-1}$ or extended mode between 100 and $3200 \mathrm{~cm}^{-1}$. The spectra were obtained under a range of laser power conditions whereby the power was varied to confirm that the peaks obtained in the data presented avoid photodamage and are reproducible. The SERS spectra presented in this article have been background corrected using a polynomial multipoint fitting function (WiRe software, Renshaw).

\section{RESULTS AND DISCUSSION}

Following evaporation of a $5 \mathrm{~nm}$ layer of chrome for adhesion the inverted pyramid substrates had a $100 \mathrm{~nm}$ layer of gold evaporated by (i) thermal and (ii) electron-beam evaporation. Our strategy was to provide surfaces that are as smooth and with as little background SERS signal as possible. The surfaces were imaged at high resolution using a HIM; the surface roughness of both the flat surface and the inverted pyramid faces was shown to be sub-50nm and of equivalent texture on the flat top surface and within the inverted pyramid regions as can be seen in Figure 3.

The chosen inverted pyramid dimensions are optimal for our proposed SERS studies using $785 \mathrm{~nm}$ excitation light, as assessed previously.[6] Raman spectra were obtained using $785 \mathrm{~nm}$ laser excitation from a number of samples of the inverted pyramid structures where gold was evaporated to a 
thickness of $100 \mathrm{~nm}$ by (i) thermal evaporation and (ii) electronbeam evaporation. The samples with the lowest background spectra were obtained from samples where the gold was deposited by electron beam evaporation and were used for the subsequent studies. Samples prepared by electron beam evaporation were then coated with Texas Red succinimide (Invitrogen) $(100 \mu \mathrm{l})$ at a concentration of $1 \mu \mathrm{M}$ in ethanol and then dried. A Raman spectrum obtained using $785 \mathrm{~nm}$ excitation from the region containing the pyramid pits and from the gold-coated surrounding area that was flat. For spectra taken under identical conditions it was impossible to detect the two characteristic SERS peaks at $1500 \mathrm{~cm}^{-1}$ and $1643 \mathrm{~cm}^{-1}$ for Texas Red[5] from the flat, gold-covered surrounding area, but an intense easily observed spectrum for Texas Red was obtained for the inverted pyramid region. This showed that the gold covering the flat regions was sufficiently smooth not to provide nanoscale field localization, and that the spectrum obtained from the inverted pyramid region is greatly enhanced by the plasmon resonant cavity structure.

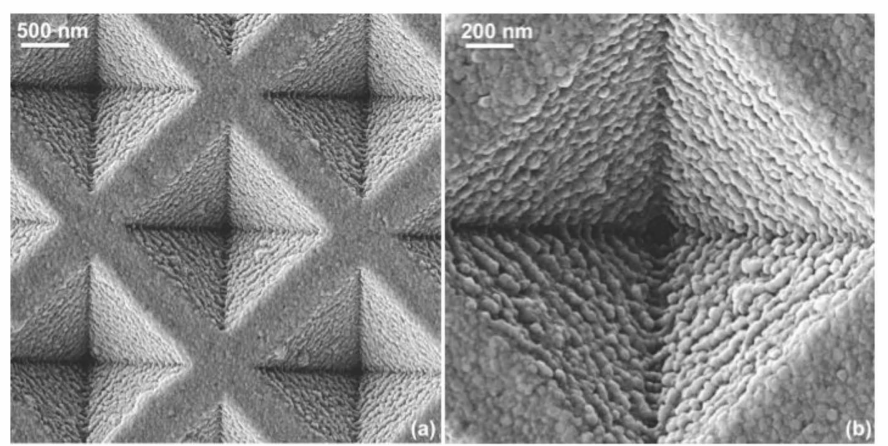

Fig. 3. (a) Helium Ion Microscope image of inverted pyramids showing the gold surface created by electron beam deposition; (b) Magnified view showing single inverted pyramid cavity.

The next step was to evaluate oligonucleotides composed of single nucleic acids $\left(\mathrm{dA}_{10} \mathrm{dC}_{10} \mathrm{dT}_{10}\right.$ and $\left.\mathrm{dG}_{10}\right)$. These were physically absorbed onto the surface of the inverted gold pyramid structure as a water solution of $(1 \mu \mathrm{M}, 100 \mu \mathrm{L})$ and Raman spectra obtained using $785 \mathrm{~nm}$ excitation. The data is presented in table II and compared against the previously reported assigned Raman Shift values. The shifts of the major peaks are similar to those previously obtained from polyribonucleotides physically absorbed on the surface of silver torus structures on a silicon/silicon dioxide substrate.

TABLE II. MAJOR RAMAN PEAKS OBTAINED FOR OLIGONUCLEOTIDES $\left(\mathrm{dA}_{1} \bullet, \mathrm{dG}_{1} \bullet \mathrm{dC}_{1} \bullet \mathrm{dT}_{1}\right)$ PHYSICALLY ABSORMED ON THE INVERTED PYRAMID STRUCTURES AS COMPARED TO LITERATURE DATA FOR RELATED STUDIES OBTAINED BY SERS METHODS

\begin{tabular}{|l|l|c|}
\hline \multirow{2}{*}{ Sample } & \multicolumn{2}{|c|}{ SERS data $\left(\mathbf{c m}^{-\mathbf{1}}\right)$} \\
\cline { 2 - 3 } & $\begin{array}{c}\text { Inverted pyramid structures } \\
\left(\mathbf{c m}^{-1}\right)\end{array}$ & $\begin{array}{c}\text { SERS Literature data } \\
\left(\mathbf{c m}^{-1}\right)\end{array}$ \\
\hline $\mathrm{dA}_{1} \bullet$ & $1337,1324,727$ & $\begin{array}{c}1320,731^{\mathrm{c}} \\
736^{\mathrm{d}}\end{array}$ \\
\hline $\mathrm{dC}_{\mathbf{1}}$ & $1274($ broad $), 787$ & $1633,1299,1023,790^{\mathrm{c}}$ \\
\hline $\mathrm{dT}_{1} \bullet$ & $1651,1372,744$ & $1360,790^{\mathrm{c}}$ \\
\hline $\mathrm{dG}_{1} \bullet$ & $1578,1481,1332,686$ & $1572,1340,688,650^{\mathrm{c}}$ \\
\hline
\end{tabular}

Probe oligonucleotides (Table I) were then coupled to the gold surface by means of a hexa-thiol linkage as outlined in the Materials and Methods section. Once the chemical attachment was complete and the surface dried, the inverted gold pyramid sample was evaluated by Raman microscopy $(785 \mathrm{~nm})$. The resulting spectrum contains a large number of sharp peaks which are assigned in Figure 4 (a), based upon the data obtained for the mono-oligonucleotides (Table II). As a check the flat gold surface was also evaluated, but no spectra could be obtained using the same conditions. The Texas red label was incorporated to provide a simple approach to confirm the attachment of the DNA by fluorescence microscopy and also as a potential probe for the penetration of the plasmon field into the DNA sequence as the Texas Red at the 3'-end of the DNA would be expected to be most remote from the gold surface. DNA-oligonucleotide functionalized inverted pyramid samples prepared in a similar manner were then hybridized with the complementary target sequence (Table 1) and the inverted pyramid regions also evaluated by Raman microscopy. The resulting spectrum is presented in Figure 4 (b) with the peaks tentatively assigned against literature data.
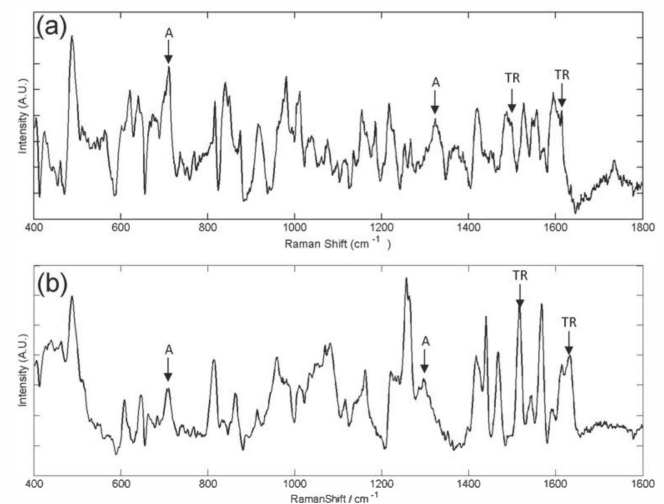

Fig. 4. SERS of DNA oligonucleotides. Peaks assigned to adenine (A) and Texas red (TR) are identified in both spectra (a) probe sequence (Table I) attached to the surface of the gold inverted pyramid substrate; (b) hybridized target sequence (Table I) to a similarly prepared sample as shown in (a).

The oligonucleotide used is estimated to be $7.5 \mathrm{~nm}$ long, thus we anticipate that all the DNA oligonucleotides would all experience the field of the plasmons. Our results obtained demonstrate that SERS spectra can be easily achieved with these gold inverted pyramid structures. It is clear from the data shown in Figure 4 that we obtain a large number of SERS peaks for the probe and hybridized target samples. Although it is possible to see the peaks assigned to SERS resonances for the adenine bases, these bases are in low abundance in the sequence (Table I). Other peaks could be tentatively asigned for the other nucleic acid bases, however it is our view and as seen by others,[10] the SERS resonances shift slightly for nucleic acid bases when hybridized. Thus a more direct approach for the detection of specific DNA sequences using these inverted pyramid substrates now requires an extensive study of multiple DNA sequences, the creation of a database of SERS data and subsequent principal component analysis methods for pattern recognition in the SERS data of subsequent unidentified sequences.[11] 
Although quantitation is a requirement for many analytical methods, SERS techniques present a number of issues that need to be considered. Molecules must be close to the metal surface because most SERS enhancement comes from the first monolayer on the surface. For oligomeric molecules, such as DNA, the surface density of molecules needs consideration as at high densities self-absorption and the scattering efficiency will be affected as a result of differences in the alignment of the bases in the local field. In addition to this the SERS cross section of each of the nucleic acid bases is different, thus SERS approaches are best suited for DNA sequence detection. So although these inverted pyramid structures are ideal for the very sensitive detection of analytes,[10] all DNA-based analytes need to be calibrated on a case by case basis to obtain an accurate quantification. However, what is clear is that DNA sequences are easily detected in these gold inverted pyramid structures and that there is much potential for the development of rapid DNA-based diagnostic assays in this format.

\section{CONCLUSION}

In conclusion, direct detection of DNA sequences is very easily achieved by SERS using the inverted gold pyramid structures which have been specially fabricated with very smooth gold surfaces to facilitate efficient plasmon resonant coupling within the inverted pyramid cavity. This research is on-going with a view to detect a range of DNA modifications and low copy numbers of DNA.

\section{ACKNOWLEDGMENT}

We acknowledge the BBSRC (Ref: BB/I023720/1) and the CARIPLO Foundation for funding and Renishaw Diagnostics for providing uncoated Klarite ${ }^{\circledR}$ samples. We also acknowledge Dr Stuart Boden for help obtaining the HIM images of the samples.

\section{REFERENCES}

[1] G. McNay, D. Eustace, W. E. Smith et al., "Surface-Enhanced Raman Scattering (SERS) and Surface-Enhanced Resonance Raman Scattering (SERRS): A Review of Applications," Applied Spectroscopy, vol. 65, no. 8, pp. 825-837, Aug, 2011.

[2] A. Barhoumi, and N. J. Halas, "Detecting Chemically Modified DNA Bases Using Surface-Enhanced Raman Spectroscopy," Journal of Physical Chemistry Letters, vol. 2, no. 24, pp. 31183123, Dec 15, 2011.

[3] J. P. Camden, J. A. Dieringer, J. Zhao et al., "Controlled Plasmonic Nanostructures for Surface-Enhanced Spectroscopy and Sensing," Accounts of Chemical Research, vol. 41, no. 12, pp. 1653-1661, Dec, 2008

[4] M. Green, F. M. Liu, L. Cohen et al., "SERS platforms for high density DNA arrays," Faraday Discussions, vol. 132, pp. 269-280, 2006, 2006.

[5] S. Mahajan, J. Richardson, T. Brown et al., "SERS-Melting: A New Method for Discriminating Mutations in DNA Sequences," Journal of the American Chemical Society, vol. 130, no. 46, pp. 15589-15601, Nov 19, 2008.

[6] N. M. B. Perney, J. J. Baumberg, M. E. Zoorob et al., "Tuning localized plasmons in nanostructured substrates for surfaceenhanced Raman scattering," Optics Express, vol. 14, no. 2, pp. 847-857, Jan 23, 2006

[7] M. F. A. Muttalib, S. Z. Oo, and M. D. B. Charlton, "Experimental measurement of photonic/plasmonic crystal dispersion, applied to the investigation of surface Plasmon dispersion for SERS sensing applications," Integrated Optics: Devices, Materials, and Technologies Xvi, Proceedings of SPIE J. E. Broquin and G. N. Conti, eds., 2012.

[8] S. Z. Oo, M. D. B. Charlton, D. Eustace et al., "Optimization of SERS enhancement from nanostructured metallic substrate based on arrays of inverted rectangular pyramids and investigation of effect of lattice non-symmetry," Plasmonics in Biology and Medicine Ix, Proceedings of SPIE T. VoDinh and J. R. Lakowicz, eds, 2012.

[9] S. Z. Oo, M. D. B. Charlton, M. E. Pollard et al., "3D analysis of surface Plasmon dispersion for SERS sensor based on inverted pyramid nanostructures," Photonic and Phononic Properties of Engineered Nanostructures $I$, Proceedings of SPIE A. Adibi, S. Y. Lin and A. Scherer, eds., 2012.

[10] N. M. B. Perney, F. J. G. de Abajo, J. J. Baumberg et al., "Tuning localized plasmon cavities for optimized surface-enhanced Raman scattering," Physical Review B, vol. 76, no. 3, Jul, 2007.

[11] D. Van de Sompel, E. Garai, C. Zavaleta et al., "A Hybrid Least Squares and Principal Component Analysis Algorithm for Raman Spectroscopy," Plos One, vol. 7, no. 6, Jun 18, 2012. 